\title{
Comparing histologic evaluation of prostate tissue using nonlinear microscopy and paraffin H\&E: a pilot study
}

\author{
Lucas C. Cahill ${ }^{1,2} \cdot$ James G. Fujimoto ${ }^{2} \cdot$ Michael G. Giacomelli ${ }^{2} \cdot$ Tadayuki Yoshitake $^{2} \cdot$ Yubo Wu $^{3} \cdot$ Douglas I. Lin $^{3}$. \\ Huihui $\mathrm{Ye}^{3} \cdot$ Oscar M. Carrasco-Zevallos ${ }^{2} \cdot$ Andrew A. Wagner $\mathbb{C}^{4} \cdot$ Seymour Rosen $^{3}$
}

Received: 8 October 2018 / Revised: 27 November 2018 / Accepted: 28 November 2018 / Published online: 26 March 2019

(c) United States \& Canadian Academy of Pathology 2019

\begin{abstract}
Rapid histological assessment of large areas of prostate tissue is required for many intraoperative consultation scenarios such as margin evaluation. Nonlinear microscopy (NLM) enables imaging of large (whole mount) specimens without freezing or cryotoming. This study demonstrates rapid histological imaging of unsectioned prostate cancer surgical specimens using nonlinear microscopy and compares features of prostate pathology to standard paraffin embedded H\&E histology. Fresh or formalin fixed specimens were stained in $2.5 \mathrm{~min}$ with fluorescent nuclear and stromal dyes. Nonlinear microscopy images of unsectioned tissues were generated by nonlinear (two-photon) excitation of the fluorophores, where fluorescence is only emitted from tissue at the microscope focus, avoiding the need for physical sectioning. The images were displayed in real time using a color scale similar to H\&E, then tissues were processed for standard paraffin embedded H\&E histology. Seventy nonlinear microscopy and corresponding paraffin H\&E images of fresh and fixed prostate specimens (15 cancer, 55 benign) from 24 patients were read by genitourinary pathologists to assess if nonlinear microscopy could achieve an equivalent evaluation to paraffin embedded H\&E histology. Differences between nonlinear microscopy images and paraffin $\mathrm{H} \& \mathrm{E}$ slides, including cytoplasmic color and stromal density, were observed, however nonlinear microscopy images could be interpreted with minimal training. Nonlinear microscopy enabled visualization of benign, atrophic and hyperplastic glands and stroma, ejaculatory ducts, vasculature and inflammatory changes. Nonlinear microscopy enabled identification of typical and variants of adenocarcinoma, as well as Gleason patterns. Perineural invasion and extraprostatic extension could also be assessed. Nonlinear microscopy images closely resemble paraffin H\&E slides and enable rapid assessment of normal prostate architecture, benign conditions, and carcinoma in freshly excised and fixed specimens. Nonlinear microscopy can image large regions of tissue, equivalent to multiple frozen section tissue blocks, within minutes because cryotoming/ microtoming are not required, making it a promising technique for intraoperative consultation.
\end{abstract}

Seymour Rosen

srosen@bidmc.harvard.edu

1 Harvard-MIT Division of Health Sciences and Technology, Harvard Medical School and Massachusetts Institute of Technology, Cambridge, MA, USA

2 Department of Electrical Engineering and Computer Science and Research Laboratory of Electronics, Massachusetts Institute of Technology, Cambridge, MA, USA

3 Department of Pathology, Beth Israel Deaconess Medical Center, Harvard Medical School, Boston, MA, USA

4 Department of Surgery, Division of Urology, Beth Israel Deaconess Medical Center, Harvard Medical School, Boston, MA, USA

\section{Introduction}

Rapid histological assessment of prostate tissue may have applications to intraoperative consultation scenarios such as surgical margin evaluation [1-3]. Optical techniques, including structured illumination microscopy $[4,5]$, optical coherence tomography [6-9], light sheet microscopy [10] and nonlinear microscopy (NLM) [11], enable rapid histological imaging of freshly excised tissues without requiring physical cryotome or microtome sectioning. Nonlinear microscopy generates images in unsectioned tissue by using a short pulse laser beam which is scanned across the tissue and generates nonlinear (two-photon) excitation of exogenous fluorescent stains. Fluorescence is only emitted from a few micron thick slice of tissue at the laser beam focus, avoiding the need for physical sectioning [12]. Nonlinear microscopy images 
closely resembling $\mathrm{H} \& \mathrm{E}$ slides can be generated using fluorescent nuclear and stromal/cytoplasmic stains, displaying the fluorescence signals with H\&E colors. Because physical sectioning is not required, large specimens can be rapidly imaged without the freezing and cryotoming required for frozen section analysis. We have demonstrated that nonlinear microscopy can be used to evaluate fresh breast tissue surgical specimens [13-15]. Other groups have used nonlinear microscopy to identify prostate architecture in both rat $[16,17]$ and human [18], however, nonlinear microscopy has not been used to evaluate fresh prostate tissue with nuclear and stromal/cytoplasmic contrast generating images analogous to paraffin H\&E slides. Here, we describe a method for preparing and imaging centimeter-scale, fresh prostate tissue in minutes using nonlinear microscopy with exogenous fluorescent contrast agents and virtual $\mathrm{H} \& \mathrm{E}$ color display. We compare nonlinear microscopy imaging of fresh and fixed, benign and malignant prostate tissue with paraffin embedded H\&E slides.

\section{Materials and methods}

\section{Tissue preparation}

Seventy specimens (15 cancer, 55 benign) of fresh and fixed prostate tissue (Table 1) were collected from 24 patients who underwent a prostatectomy. All research was performed according to protocols approved by Beth Israel Deaconess Medical Center Committee on Clinical Investigations and Institutional Review Board and Massachusetts Institute of Technology Committee on the Use of Humans as Experimental Subjects. Informed consent was waived by both committees.

Fresh and fixed tissue specimens were stained in acridine orange $(40 \mu \mathrm{g} / \mathrm{ml})$ and sulforhodamine 101 (SR101 $40 \mu \mathrm{g} / \mathrm{ml})$ in a 1:1 ethanol:water solution for $2 \mathrm{~min}$ and rinsed for $30 \mathrm{~s}$ in phosphate-buffered saline (Fig. 1a). Acridine orange provides nuclear contrast, analogous to hematoxylin, while sulforhodamine 101 provides stromal and cytoplasmic contrast analogous to eosin. These fluorescent stains are advantageous because they rapidly penetrate fresh tissue, can be removed to undetectable levels with standard histology solvents [14], and can be excited at a wide range of wavelengths, making them amenable to nonlinear microscopy. Unsectioned tissue was

Table 1 Summary of prostate tissue specimens evaluated using nonlinear microscopy and corresponding paraffin $\mathrm{H} \& \mathrm{E}$

\begin{tabular}{llcc}
\hline Specimens (patients) & Fresh & Fixed & Total \\
\hline Cancer & $13(8)$ & $2(2)$ & $15(10)$ \\
Benign & $24(7)$ & $31(7)$ & $55(14)$ \\
\hline
\end{tabular}

MP-2018-0876 placed on a glass coverslip and compressed with biopsy foam to provide a flat imaging surface.

\section{Image acquisition and processing}

Nonlinear microscopy was first used to image the unsectioned prostate tissue in real-time (Fig. 1b), by translating the specimen in the $\mathrm{x}-\mathrm{y}$ plane, with interchangeable magnification objectives, analogously to the way a traditional light microscope is used to view slides [11]. The specimen $\mathrm{z}$ axis was actuated up and down to focus at different depths up to $100 \mu \mathrm{m}$ below the prostate tissue surface (enabling serial sectioning without having to physically section). H\&E color display was adjustable to suit user preference. This method can enable rapid evaluation of margins as well as focal pathology, since specific regions of interest can be quickly selected and examined under operator control.

A nonlinear microscopy image of the entire tissue cross section was also acquired by imaging multiple high magnification overlapping regions (Fig. 1c), at or slightly below the tissue surface. The frames were stitched using Microsoft Image Composite Editor. This produces a digital archive image which can be viewed analogously to a whole slide image generated by a slide scanner. Operating in this scanning mode requires significant delay in evaluation and is not feasible for real-time analysis, but enables comparison of nonlinear microscopy images with paraffin embedded H\&E as well as archiving and assessment by multiple readers.

Nonlinear microscopy images were acquired using either a 10x, 0.45 numerical aperture (NA) air objective (CFI Plan Apo Lambda, Nikon) or a 20x, 1.0 NA water immersion objective (XLUMPFL20XW 1.0 NA, Olympus) in one of three microscope configurations previously described $[11,14]$ and shown in Table 2. In all configurations, a $590 \mathrm{~nm}$ high-pass dichroic beam splitter split the fluorescent emission light through two emission filters (ET540/40 m, Chroma Technology and FF01-650/60, Semrock) onto two photomultiplier tubes (Hamamatsu) to detect the acridine orange and sulforhodamine 101 fluorescence.

After nonlinear microscopy imaging, the fresh prostate tissue specimens were fixed in formalin for a minimum of $12 \mathrm{~h}$ on the glass coverslip to maintain orientation and limit tissue shrinkage. Both the tissue specimens that were imaged fresh and fixed by nonlinear microscopy were then processed for standard paraffin embedded histology by pressing the imaged face into the embedding mold to maintain specimen flatness, microtomed into $5 \mu \mathrm{m}$ sections, and stained with hematoxylin and eosin. The paraffin H\&E slides were scanned with a digital slide scanner (20x magnification, Aperio) then the image size was scaled and rotated to match the nonlinear microscopy images and correct for minor shrinkage due to dehydration and paraffinization. 
A Nonlinear microscopy (NLM) of tissue without microtome or cryotome sectioning

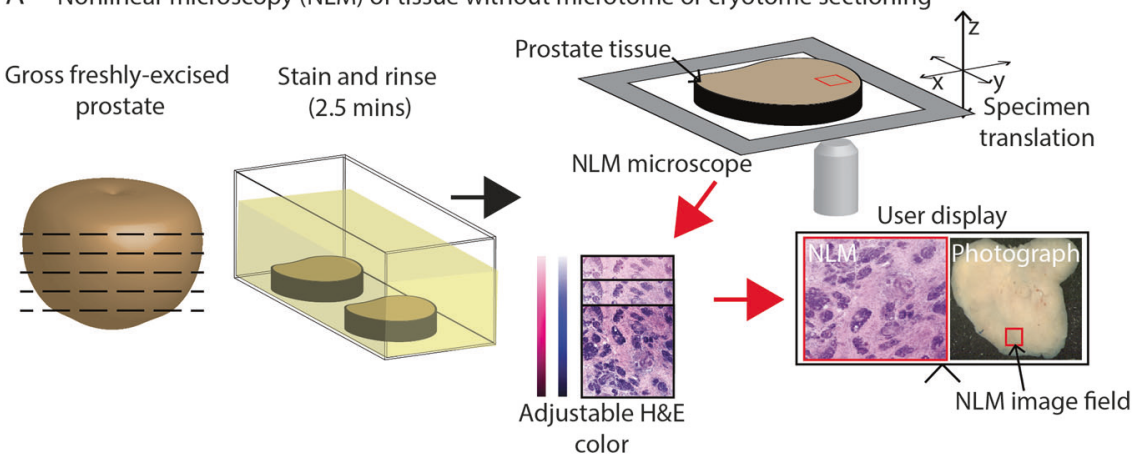

B Real-time evaluation of regions of interest
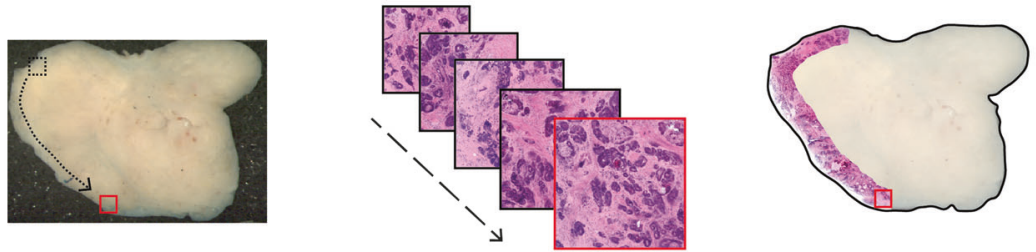

User display updated in real time

C NLM imaging of entire tissue face for digital archiving
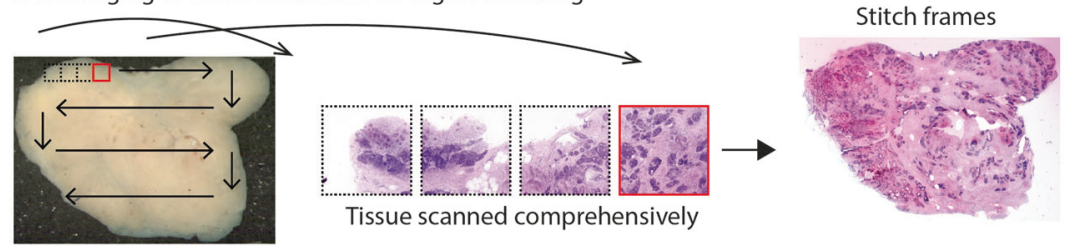

Fig. 1 a The prostate specimens were transected, cross sections were rapidly stained with acridine orange and sulforhodamine 101, rinsed and then placed on the nonlinear microscope stage. 10x and $20 \mathrm{x}$ objectives were used to visualize the tissue. The tissue was translated in the $\mathrm{x}, \mathrm{y}$, and $\mathrm{z}$ direction to examine the entire cross section of tissue (whole mount) up to $100 \mu \mathrm{m}$ in depth (analogous to serial sectioning). The H\&E color display was generated using the acridine orange and sulforhodamine 101 fluorescence signals. The user display showed a photograph (right side) of the prostate cross section and a high magnification nonlinear microscopy (NLM) image (left side) of a user selected region of interest (marked in red). b For real time intraoperative consultation, the user can translate the specimen in the $x-y$ direction to rapidly view different regions and change the focus in the $z$ direction to view different depths. The images can be recorded to create a digital record. c Nonlinear microscopy can also automatically image the entire specimen cross section by stitching multiple high magnification fields. This operating mode is too time consuming for intraoperative consultation, but is useful for digital archiving purposes (analogous to slide scanning) and for comparing nonlinear microscopy images to standard paraffin embedded H\&E slides
Table 2 Nonlinear microscope configurations

\begin{tabular}{llll}
\hline $\begin{array}{l}\text { Objective lens mag. } \\
\text { (immersion) }\end{array}$ & $\begin{array}{l}\text { Frame size } \\
(\mathrm{mm})\end{array}$ & $\begin{array}{l}\text { Pixel size } \\
(\mu \mathrm{m})\end{array}$ & $\begin{array}{l}\text { Excitation } \\
\text { wavelength }(\mathrm{nm})\end{array}$ \\
\hline 20x (water) & $\begin{array}{l}0.512 \times \\
0.512\end{array}$ & 0.5 & 780 \\
& $\begin{array}{l}1.08 \times 1.08 \\
\text { 10x (air) }\end{array}$ & 0.75 & 780 \\
$10 \mathrm{x}$ (air) & $\begin{array}{l}2.048 \times \\
2.048\end{array}$ & 1 & 1030 \\
& & & \\
\hline
\end{tabular}

MP-2018-0876-R

\section{Tissue evaluation}

Three genitourinary pathologists evaluated H\&E slides and nonlinear microscopy images in an unblinded observational reading. The main analysis was done by SR, who has over 25 years of experience. Nonlinear microscopy digital images were viewed in OpenSeadragon, a web-based viewer that enables variable magnification review. Paraffin H\&E slides or scanned slides were evaluated using a histology microscope or with OpenSeadragon. Similarities and differences between nonlinear microscopy and paraffin $\mathrm{H} \& \mathrm{E}$ were characterized.

\section{Results}

Nonlinear microscopy enables visualization of normal prostate architecture, benign conditions, and prostate carcinoma. 

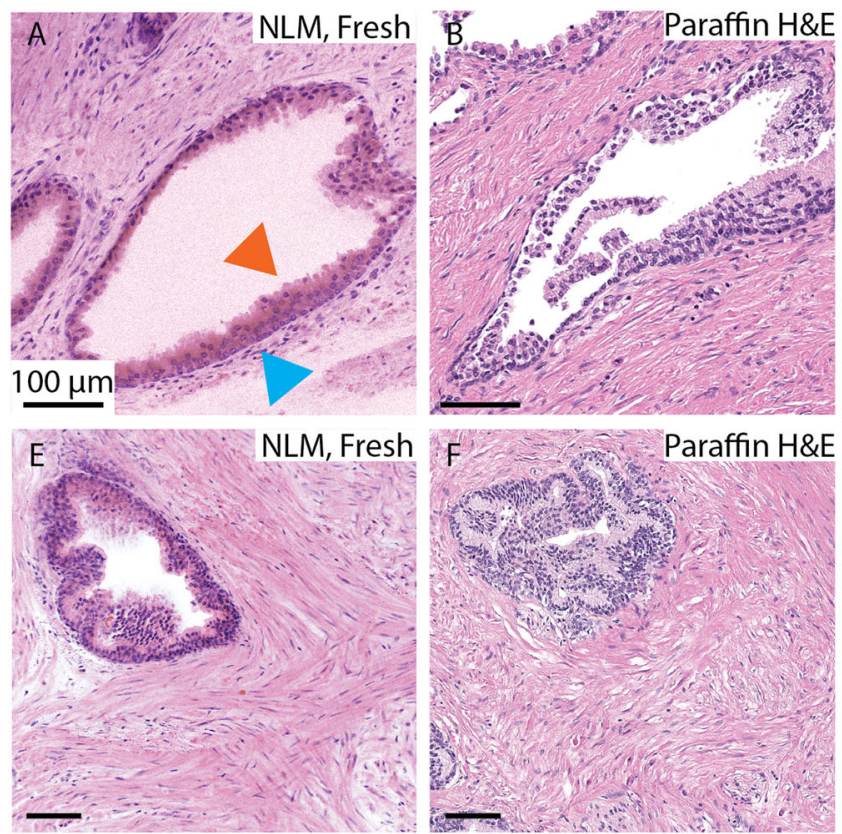

Fig. 2 Examples of prostate tissue architecture visualized using nonlinear microscopy imaging of freshly excised, unfixed specimens that were not physically sectioned versus corresponding standard paraffin embedded H\&E slides after standard histological processing. a Nonlinear microscopy and $\mathbf{b}$ paraffin $H \& E$ of benign ducts with basal (blue arrow) and secretory (orange arrow) layers (20x objective), c nonlinear

\section{Prostate architecture}

Examples of prostate tissue architecture acquired using nonlinear microscopy on freshly excised, unfixed specimens and corresponding paraffin $\mathrm{H} \& \mathrm{E}$ slide images are shown in Fig. 2. An outer layer of flattened cuboidal basal cells (blue arrow) and an inner secretory layer (orange arrow) of columnar cells with abundant cytoplasm and mainly uniform nuclei positioned basally are clearly visualized in benign ducts (Fig. 2a, b). Neural bundles with ganglion cells, with their abundant cytoplasm, appear similar in nonlinear microscopy images (Fig. 2c) and paraffin $\mathrm{H} \& \mathrm{E}$ slides (Fig. 2d). The appearance of stroma in nonlinear microscopy images of freshly excised, unfixed tissue (Fig. 2e) is denser than that of paraffin H\&E sections (Fig. 2f). The fixation, dehydration, and physical sectioning required to obtain a paraffin $\mathrm{H} \& \mathrm{E}$ slide is most likely responsible for these differences. Additional differences between nonlinear microscopy and paraffin H\&E include the prominent pink appearance of inner elastic lamina of arteries in nonlinear microscopy (Fig. 2g) that is not present on paraffin H\&E (Fig. 2h).

A comparison of normal prostate architecture visualized by nonlinear microscopy imaging of fixed tissue that was not processed or microtomed for paraffin histology versus standard H\&E stained paraffin sections is shown in Fig. 3.
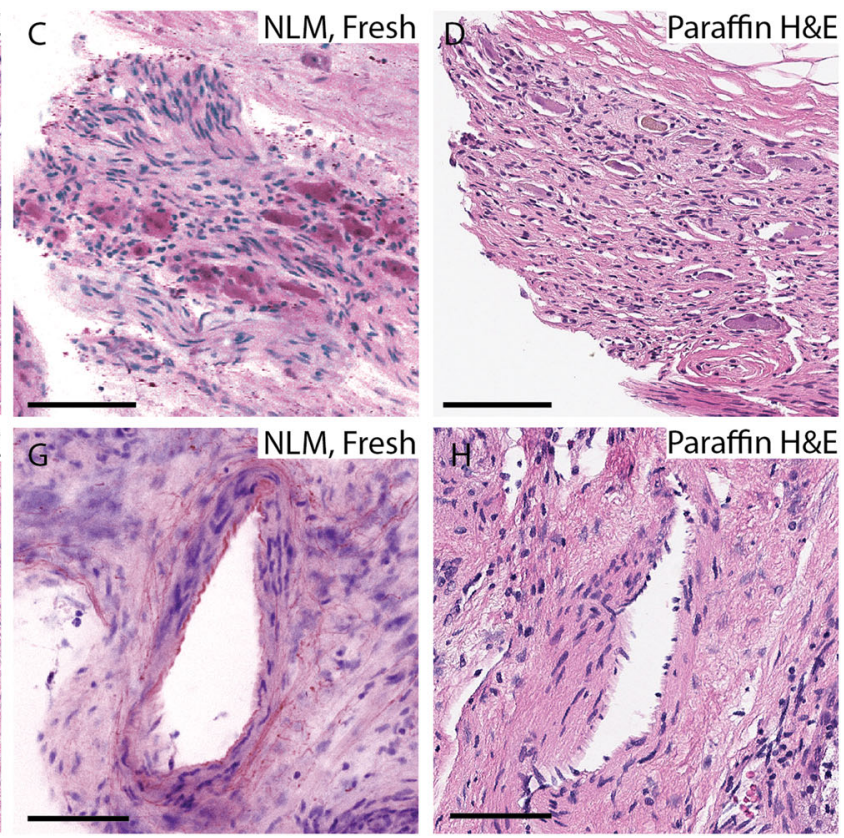

microscopy, and d paraffin $\mathrm{H} \& \mathrm{E}$ of a neural bundle (10x objective, $0.75 \mu \mathrm{m}$ pixel), e nonlinear microscopy of stroma (10x objective, $0.75 \mu \mathrm{m}$ pixel) with $\mathbf{f}$ corresponding paraffin $\mathrm{H} \& \mathrm{E}$, and $\mathbf{g}$ nonlinear microscopy and $\mathbf{h}$ paraffin $\mathrm{H} \& \mathrm{E}$ of an artery (10x objective, $1 \mu \mathrm{m}$ pixel). All scale bars are $100 \mu \mathrm{m}$

An artery (Fig. 3a, b), characterized by its spindle shaped smooth muscle cells with elongated nuclei, is shown alongside a vein. The lumens of these vessels are filled with red blood cells. In typical analysis of paraffin sections using an ordinary microscope, colors other than the pink and blue from hematoxylin and eosin are seen. Nonlinear microscopy images are generated using only two discrete fluorescent color channels, so the red blood cells do not appear red as they do in the paraffin H\&E sections, and instead appear pink. Ejaculatory ducts display characteristic mucosal folds and pseudostratified columnar epithelium in nonlinear microscopy (Fig. 3c) matching that of the corresponding paraffin H\&E (Fig. 3d). Of note, nonlinear microscopy images of fixed tissue that was not processed or microtomed for paraffin histology did not have apparent differences from nonlinear microscopy images of freshly excised, unfixed specimens. The use of fixed tissue increased tissue availability and enabled us to assess the possibility of using nonlinear microscopy for examining smaller tissue fragments (core biopsies) which could be fixed while being imaged.

\section{Benign conditions}

Nonlinear microscopy images of fixed prostate tissue that was not processed or microtomed for paraffin histology and 


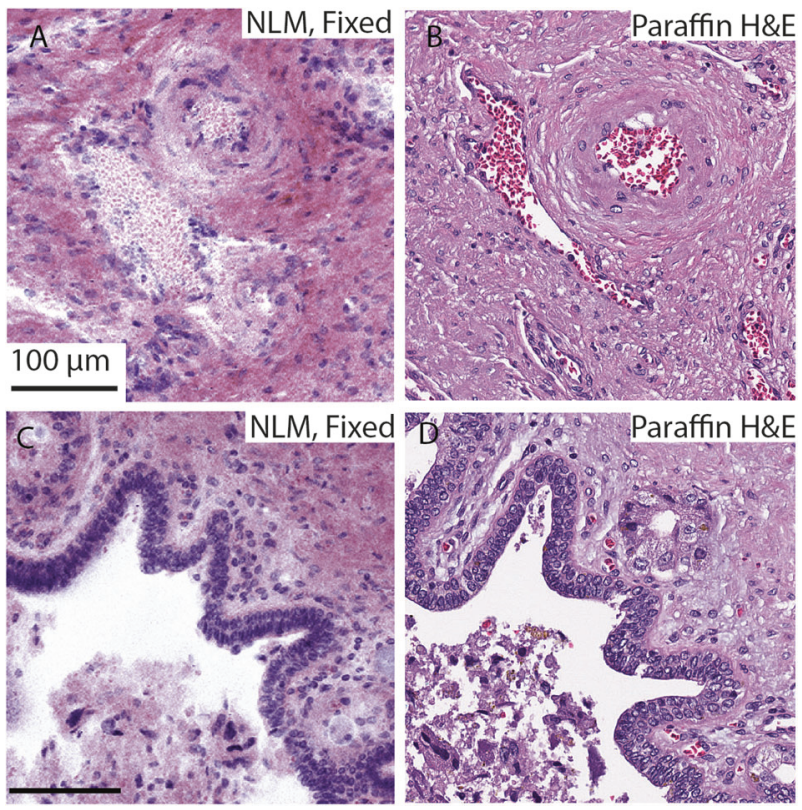

Fig. 3 Examples of prostate tissue architecture visualized by nonlinear microscopy imaging of fixed tissue that was not processed or physically sectioned versus corresponding standard paraffin embedded $\mathrm{H} \& \mathrm{E}$ slides. An artery and vein (a and b) and ejaculatory ducts (c and $\mathbf{d})$ are shown. Scale bars are $100 \mu \mathrm{m}$. (10x objective, $0.75 \mu \mathrm{m}$ pixel)

the corresponding paraffin H\&E slide from a patient who underwent Bacillus Calmette-Guerin therapy is shown in Fig. 4. Necrotizing granulomatous inflammation is apparent in both the nonlinear microscopy and paraffin H\&E with central regions of necrosis, giant cells (yellow arrow), and palisading histiocytes (red box). Benign, atrophic singlelayer basophilic glands, as indicated by the flattened epithelium, decreased cytoplasmic volume and dilated lumen, are seen throughout the tissue (blue arrow).

Nonlinear microscopy images of freshly excised, unfixed prostate tissue with benign prostatic hyperplasia and the corresponding paraffin H\&E slides are shown in Fig. 5. Stromal and glandular hyperplasia is clearly visualized in nonlinear microscopy with nodules made up of complex benign glands with luminal papillary projections throughout the tissue (red, blue).

\section{Carcinoma}

Differentiating prostate carcinoma versus benign conditions is important for both histologic diagnosis and tumor margin evaluation. Nonlinear microscopy enables visualization of prostate carcinoma with some features closely resembling those of paraffin H\&E and other features that differ, but are recognizable. Discriminating between cancerous and benign glands requires appreciation of cellular aspects such as nuclear size and nucleoli, while determining the histologic grade (Gleason score) is more reliant on visualization of glandular architectural features.

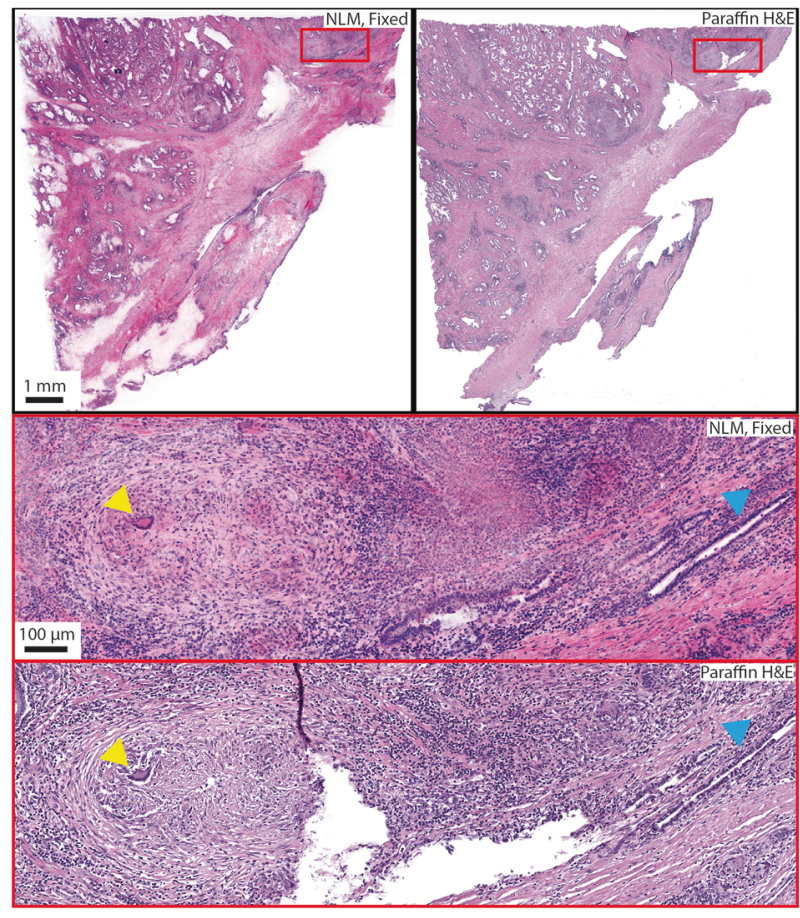

Fig. 4 A prostate specimen from a patient who underwent Bacillus Calmette-Guerin therapy. The characteristic histological patterns of this therapy are evident in nonlinear microscopy images of the fixed, unsectioned specimen and corresponding paraffin embedded $\mathrm{H} \& \mathrm{E}$ slide, including granulomatous inflammation (red) with giant cells (yellow arrow) and atrophic glands (blue arrow). (10x objective, $0.75 \mu \mathrm{m}$ pixel)

An example of a nonlinear microscopy image of fixed prostate tissue that was not processed or sectioned for paraffin histology and a corresponding paraffin H\&E slide from a patient with typical adenocarcinoma (Gleason 7 $(4+3))$ is shown in Fig. 6. Prominent nucleoli of enlarged nuclei are evident on the nonlinear microscopy images (orange box), however, the color of the cytoplasm in the nonlinear microscopy images is different than of the paraffin H\&E slide. Despite these differences, it is clear in the nonlinear microscopy image that the dominant tumor component of this prostate section is composed of sheets and cribriform patterns of fused, infiltrative glands consistent with a Gleason pattern 4 which matches that of the paraffin H\&E slide (blue, yellow). Well-formed, infiltrating glands of Gleason pattern 3 make up a minority of this carcinoma (red). Benign, atrophic glands can be seen at the periphery of the tumor.

The foamy gland variant of prostate cancer is characterized by abundant foamy cytoplasm due to numerous intracytoplasmic vacuoles. This characteristic foamy appearance is not as prominent in nonlinear microscopy images of freshly excised, unfixed tissue and instead the cytoplasm can appear eosinophilic and even amphophilic, as shown in Fig. 7. This is probably due to a combination of 




Fig. 5 Nonlinear microscopy images of a freshly excised, unfixed specimen with benign prostatic hyperplasia and corresponding paraffin
H\&E slide. Complex hyperplastic glands are abundant throughout the tissue (red and blue box). (10x objective, $0.75 \mu \mathrm{m}$ pixel) factors including insufficient rinsing of the fresh tissue, differences in nonlinear microscopy image color display versus transmission microscope visualization of paraffin $\mathrm{H} \& \mathrm{~S}$ slides, differences in specificity of fluorophore uptake in fresh tissue versus fixed tissue, and differences in specificity of acridine orange versus hematoxylin. Other features of typical adenocarcinoma, such as prominent nucleoli and enlarged nuclei are less apparent in the foamy variant as seen in both the nonlinear microscopy image of fresh tissue and paraffin $\mathrm{H} \& \mathrm{E}$ slides. In both nonlinear microscopy and paraffin $\mathrm{H} \& \mathrm{E}$, we can see glands made up of a single layer of cells with smaller, basally located nuclei with abundant cytoplasm (red, blue). Further, intraluminal crystalloids (yellow arrows), which are typically pink and rhomboid in shape in paraffin $H \& E$ slides, are present in the nonlinear microscopy images, however, they appear in multiple different colors. The architectural pattern in the nonlinear microscopy images and paraffin H\&E slides are consistent and show a foamy cell variant adenocarcinoma invading the fibromuscular tissue with a Gleason score of $6(3+3)$.

A nonlinear microscopy image of an entire cross-section (whole mount) of a freshly excised, unfixed prostate specimen from a patient who underwent a radical prostatectomy is shown in Fig. 8. Typical adenocarcinoma is readily apparent in the peripheral zone of the prostate. At higher power, perineural invasion can be seen beside normal nerve fibers among the periprostatic fat indicating extra-prostatic extension. A centrally located ganglion cell can be seen in a nerve fiber bundle with prominent nucleoli.

Figure 9 shows an example demonstrating nonlinear microscopy of freshly excised, unfixed prostate tissue that was subsequently processed for paraffin embedded H\&E and used for post-operative diagnostic purposes. Nonlinear microscopy was performed in real-time as described in the methods section (Fig. 1b) in order to rapidly identify regions of interest and demonstrate the speed required for 
Fig. 6 A nonlinear microscopy image of a fixed, unsectioned prostate specimen and corresponding paraffin $\mathrm{H} \& \mathrm{E}$ slide from a patient with typical adenocarcinoma displaying Gleason pattern 4, with ductal features (blue, yellow) and 3 (red). The nuclei are enlarged with prominent nucleoli (orange box). (20x objective)
Fig. 7 A nonlinear microscopy image of a freshly excised unfixed specimen and corresponding paraffin $\mathrm{H} \& \mathrm{E}$ slide exhibiting foamy gland variant of prostate cancer with Gleason score $6(3+3)$. The cytoplasm of the cancer cells is less 'foamy' in the nonlinear microscopy images than the paraffin H\&E (red, blue). Intraluminal crystalloids (yellow arrows) are present in the images. (10x objective, $0.75 \mu \mathrm{m}$ pixel)
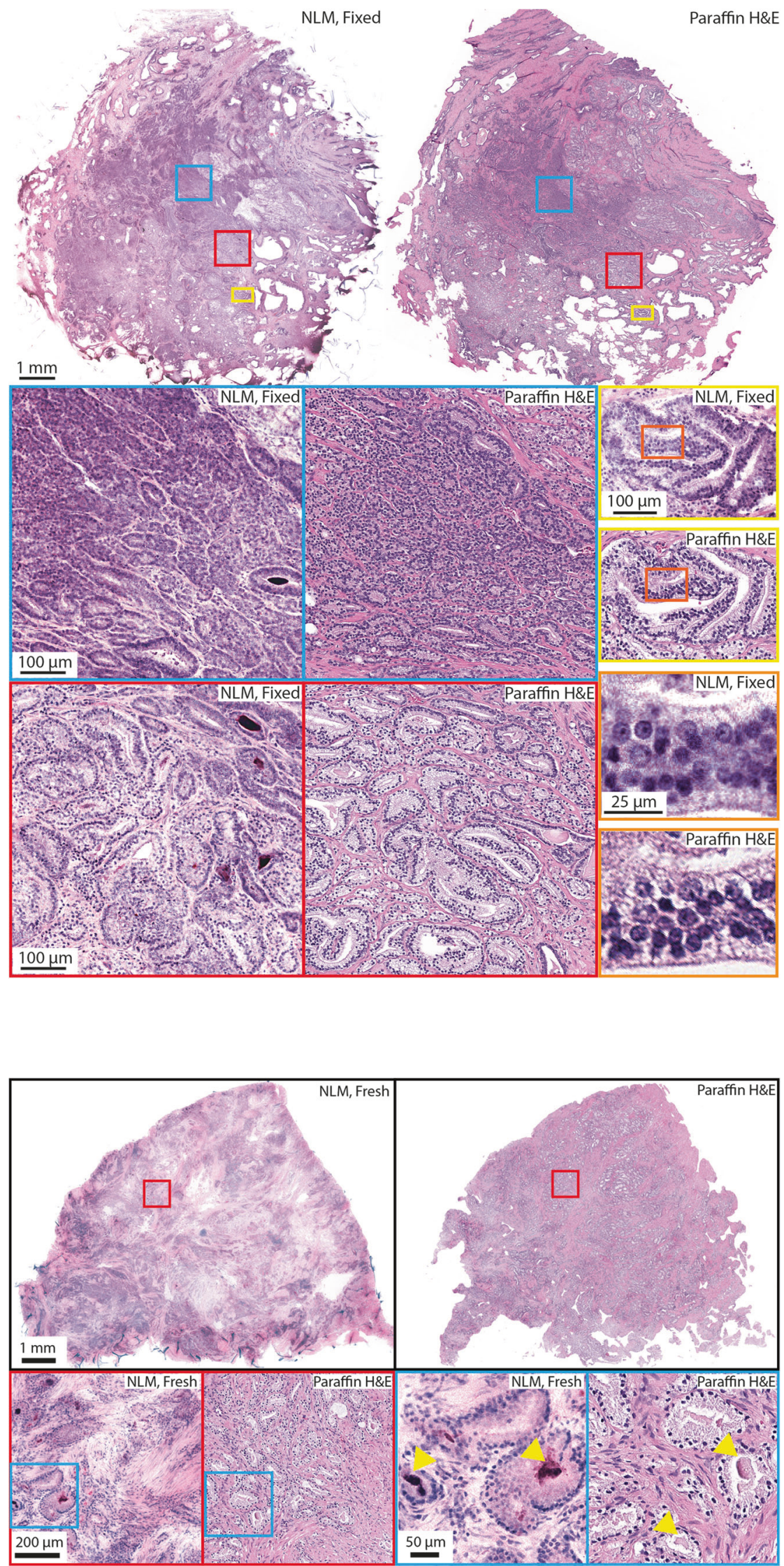


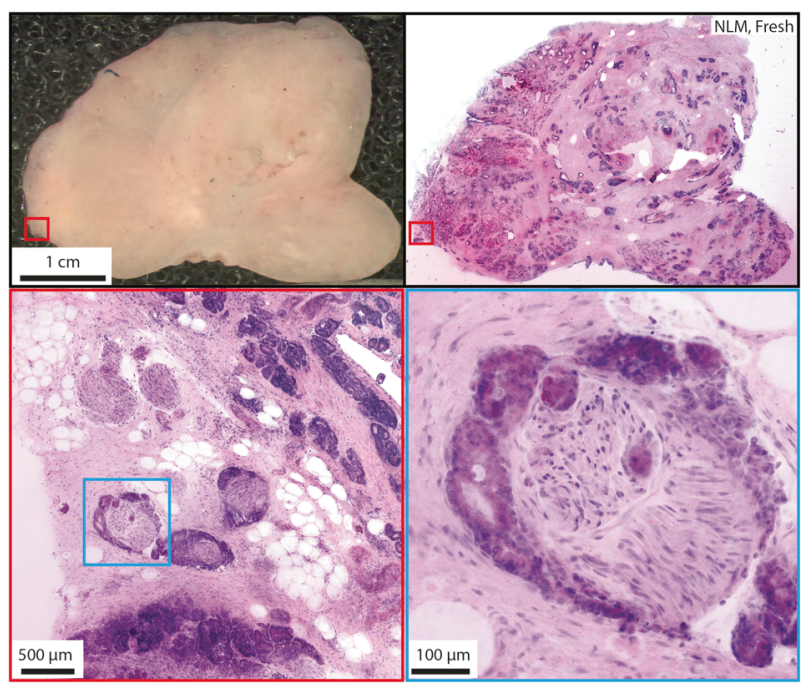

Fig. 8 A white-light photograph and nonlinear microscopy image of multi-centimeter, freshly excised, unfixed prostate tissue. Extraprostatic extension and perineural invasion is observed among the periprostatic fat (red and blue). (10x objective, $1 \mu \mathrm{m}$ pixel)

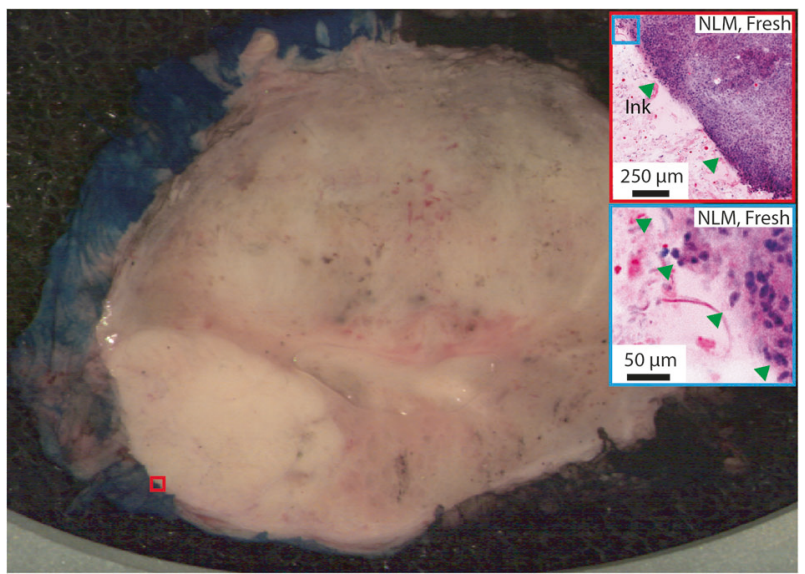

Fig. 9 A white-light photograph and nonlinear microscopy image of multi-centimeter, freshly excised, unfixed prostate tissue with tumor touching the blue ink. The tumor can be seen touching the ink in the nonlinear microscopy image (red and blue box, green arrows). The paraffin $H \& E$ slide did not show tumor touching the ink because of discrepancy between the nonlinear microscopy imaging plane and paraffin embedded H\&E section. (10x objective, $1 \mu \mathrm{m}$ pixel)

intraoperative consultation. A cancer positive margin is apparent on nonlinear microscopy (red and blue inset) and was identified with only $2.5 \mathrm{~min}$ of tissue preparation. The prostate photograph shows a grossly apparent tumor abutting the blue ink. Note that since nonlinear microscopy enables visualization of fluorescence and not true color, the ink does not appear as it does on paraffin sections and instead appears washed out with eosinophilic globules making it distinctly recognizable (green arrows). The paraffin section did not show tumor on ink, likely due to levelling of the paraffin block which introduces a discrepancy between the nonlinear microscopy imaging plane and the paraffin section plane.

\section{Discussion}

Nonlinear microscopy using rapid fluorescent stains for nuclear and stromal/cytoplasmic contrast enables prostate tissue evaluation within minutes without physical sectioning. This avoids the freezing and cryotoming required for frozen section analysis. Displaying nuclear and stromal fluorescence signals in $\mathrm{H} \& \mathrm{E}$ colors generates nonlinear microscopy images that resemble standard paraffin $\mathrm{H} \& \mathrm{E}$ histology. This study shows that nonlinear microscopy enables visualization of benign glands, vasculature, atrophic and hyperplastic glands and stroma, inflammation, and adenocarcinoma, including typical, ductal, and foamy variants. Perineural invasion and extraprostatic extension are also readily apparent. Prominent nucleoli, high nuclear to cytoplasmic ratio, and glandular and stromal architectural patterns enable prostate cancer to be recognized and Gleason patterns differentiated in nonlinear microscopy images.

Despite the similarities between nonlinear microscopy in fresh/fixed unsectioned tissue and paraffin H\&E slides, several differences were recognized. Typical histologic features that rely on color (such as bland nuclei or pale pink cytoplasm) are less apparent and consistent in nonlinear microscopy. This is because: (1) Images are generated using two discrete fluorescent color channels rather than white light transmission. Therefore, features that are not due to H\&E staining, such as the bright red appearance of red blood cells, are not apparent in nonlinear microscopy images which use nuclear and stromal fluorescence signals to synthesize H\&E-like images. (2) Freshly excised, unfixed tissue stains less homogenously than permeabilized, dehydrated tissue in paraffin sections, causing variations in appearance within and between tissues. (3) Acridine orange and sulforhodamine 101 staining in nonlinear microscopy versus hematoxylin and eosin staining of paraffin sections have similar, but not identical, staining specificities. For example, the cytoplasm of foamy variant adenocarcinoma can be darker in nonlinear microscopy images than in paraffin $H \& E$ slides.

Other than color differences between nonlinear microscopy and paraffin H\&E, freshly excised, unfixed prostate tissue and fixed tissue that was not processed or sectioned for paraffin embedded histology appears different than paraffin H\&E sections due to alcohol dehydration, xylene and paraffin infiltration, and sectioning. Alcohol dehydration, used to prepare paraffin H\&E slides, causes shrinkage and cytoplasmic retraction which can make features such as stromal fibers and nuclei more prominent. Understanding these differences enables interpretation of nonlinear 
microscopy images and facilitates feature mapping between nonlinear microscopy and paraffin H\&E.

To provide a direct comparison between nonlinear microscopy images and scanned H\&E slides, nonlinear microscopy figures (Figs. 2-7) in this paper were generated by acquiring a series of high magnification nonlinear microscopy images covering the entire tissue cross section surface (Fig. 1c), analogous to a histology slide scanner. This was convenient for digital archiving and multiple reader assessment, but is too time consuming for intraoperative applications.

It is important to note that intraoperative consultation can be performed in minutes, since comprehensive imaging of entire tissue cross sectional faces is rarely required. The edges of the tissue where surgical margins are represented as well as focal regions of interest can be rapidly assessed by translating the tissue under operator control and changing objective magnifications (Figs. 1b and 9) [11]. This operating mode would be comparable in speed to evaluating histology slides using a standard transmission light microscope, except that fresh, unsectioned tissue is imaged rather than paraffin embedded stained slides.

Since positive surgical margins occur after radical prostatectomy in an about 15\% (6.5-32\%) [19] of patients, frozen section analysis during a prostatectomy has been investigated extensively. The utility of frozen section analysis is controversial since it can be unreliable at predicting histologically positive surgical margins due to incomplete sampling [20], however, frozen section analysis protocols with more thorough sampling using multiple sections, such as those described by Schlomm et al. and Bodman et al., reduce the rate of positive surgical margins and increase the number of nerve-sparing surgeries [1-3]. These sampling techniques are time intensive and require additional infrastructure with a highly optimized workflow, including multiple cryostats, technologists, and pathologists per patient $[1,21]$. For example, even with 5 cryostats used in parallel and two pathologists and four technicians working on one patient, Schlomm, et al. report an average time of 35 minutes per patient [1].

In contrast to frozen section analysis, nonlinear microscopy does not involve freezing and cryotoming, so entire cross sections of the prostate can be rapidly processed without dissection into smaller sizes. Furthermore, multiple tissue cross sections can be stained at one time and the pathologist can begin evaluation within $2.5 \mathrm{~min}$, while frozen section analysis requires each tissue cross section to be frozen in a separate block and individually cryotomed. The simplicity and work flow advantages of nonlinear microscopy should enable more comprehensive assessment of margins than frozen section analysis in a shorter amount of time with reduced personnel requirements, facilitating wider spread adoption of intraoperative consultation in radical prostatectomy.
Furthermore, since nonlinear microscopy does not require freezing, it is non-destructive and does not have freezing artifacts. Tissue can be subsequently processed for standard paraffin embedded histology and confirmation of nonlinear microscopy findings, as demonstrated in this study. Immunohistochemistry assays (p63, Prostate Specific Antigen, NKX3.1, Cytokeratin 34 beta E12, AMACR) were tested and found to be unaffected by nonlinear microscopy processing and imaging (data not shown) as expected since the fluorescent stains are removed to undetectable levels after standard histology processing. Furthermore, nonlinear microscopy imaging can be performed up to $100 \mu \mathrm{m}$ below the tissue surface. This capability is analogous serial sectioning in histology and provides visualization of depth resolved architectural morphology which may give additional insight into pathology or image below surface contaminants such as surgical debris. Finally, nonlinear microscopy is inherently digital which enables digital archiving and may facilitate future telepathology or computer-assisted diagnostic applications.

Our study is limited because pathologists performed nonlinear microscopy evaluation unblinded to paraffin $\mathrm{H} \& \mathrm{E}$ and used a small number of specimens representing each pathology subtype. Therefore, statistical analysis was not performed. Future studies will be needed to analyze a more extensive set of prostate pathologies and assess the sensitivity and specificity of nonlinear microscopy compared to standard paraffin embedded H\&E. Both fresh and fixed specimens were used to increase tissue availability and evaluate the possibility of imaging smaller fragments such as biopsies while they are being fixed. No apparent differences between nonlinear microscopy images of fresh and fixed specimens were seen.

Nonlinear microscopy can be used to assess normal prostate architecture, benign conditions, and carcinoma in both fixed and freshly excised tissue. Images are similar to paraffin H\&E slides and imaging can be performed rapidly. Nonlinear microscopy is a promising method for future intraoperative assessment of prostate tissue, however, additional blinded studies are needed to determine its sensitivity and specificity when detecting pathology compared to the gold standard of paraffin embedded H\&E histology.

Acknowledgements This study was supported in part by the National Institutes of Health R01-CA178636-05, R01-CA075289-20, F32CA183400-02 and Air Force Office of Scientific Research AFOSR contracts FA9550-12-1-0551 and FA9550-15-1-0473.

\section{Compliance with ethical standards}

Conflict of interest JGF, MGG, TY and LCC are inventors on patent application WO2017139649: Method and apparatus for imaging 
unsectioned tissue specimens. The remaining authors declare no conflict of interest.

Publisher's note: Springer Nature remains neutral with regard to jurisdictional claims in published maps and institutional affiliations.

\section{References}

1. Schlomm T, Tennstedt P, Huxhold C, et al. Neurovascular Structure-adjacent Frozen-section Examination (NeuroSAFE) increases nerve-sparing frequency and reduces positive surgical margins in open and robot-assisted laparoscopic radical prostatectomy: experience after 11069 consecutive patients. Eur Urol. 2012;62:333-40.

2. Beyer B, Schlomm T, Tennstedt P, et al. A feasible and timeefficient adaptation of NeuroSAFE for da Vinci robot-assisted radical prostatectomy. Eur Urol. 2014;66:138-44.

3. Von Bodman C, Brock M, Roghmann F, et al. Intraoperative frozen section of the prostate decreases positive margin rate while ensuring nerve sparing procedure during radical prostatectomy. $\mathbf{J}$ Urol. 2013;190:515-20.

4. Wang M, Kimbrell HZ, Sholl AB, et al. High-resolution rapid diagnostic imaging of whole prostate biopsies using video-rate fluorescence structured illumination microscopy. Cancer Res. 2015;75:4032-41.

5. Wang M, Tulman DB, Sholl AB, et al. Gigapixel surface imaging of radical prostatectomy specimens for comprehensive detection of cancer-positive surgical margins using structured illumination microscopy. Sci Rep. 2016;6:27419.

6. Rais-Bahrami S, Levinson AW, Fried NM, et al. Optical coherence tomography of cavernous nerves: a step toward real-time intraoperative imaging during nerve-sparing radical prostatectomy. Urology. 2008;72:198-204.

7. Fried NM, Rais-Bahrami S, Lagoda GA, et al. Identification and imaging of the nerves responsible for erectile function in rat prostate, in vivo, using optical nerve stimulation and optical coherence tomography. IEEE J Sel Top Quantum Electron. 2007;13:1641-5.

8. Aron M, Kaouk JH, Hegarty NJ, et al. Second Prize: Preliminary Experience with the Niris ${ }^{\mathrm{TM}}$ Optical Coherence Tomography System during Laparoscopic and Robotic Prostatectomy. J Endourol. 2007;21:814-8.

9. D'Amico a V, Weinstein M, Li X, et al. Optical coherence tomography as a method for identifying benign and malignant microscopic structures in the prostate gland. Urology 2000;55:783-7.

10. Glaser AK, Reder NP, Chen Y, et al. Light-sheet microscopy for slide-free non-destructive pathology of large clinical specimens. Nat Biomed Eng. 2017;1:0084.

11. Giacomelli MG, Yoshitake T, Cahill LC, et al. Multiscale nonlinear microscopy and widefield white light imaging enables rapid histological imaging of surgical specimen margins. Biomed Opt Express. 2018;9:2457.

12. Denk W, Strickler J, Webb W. Two-photon laser scanning fluorescence microscopy. Science. 1990;248:73-6.

13. Tao YK, Shen D, Sheikine Y, et al. Assessment of breast pathologies using nonlinear microscopy. Proc Natl Acad Sci USA. 2014;111:15304-9.

14. Cahill LC, Giacomelli MG, Yoshitake T, et al. Rapid virtual hematoxylin and eosin histology of breast tissue specimens using a compact fluorescence nonlinear microscope. Lab Invest. 2018;98:150-60.

15. Giacomelli MG, Husvogt L, Vardeh H, et al. Virtual hematoxylin and eosin transillumination microscopy using epi-fluorescence imaging. PLoS ONE. 2016;11:e0159337.

16. Yadav R, Mukherjee S, Hermen M, et al. Multiphoton microscopy of prostate and periprostatic neural tissue: a promising imaging technique for improving nerve-sparing prostatectomy. J Endourol. 2009;23:861-7.

17. Durand M, Jain M, Aggarwal A, et al. Real-time in vivo periprostatic nerve tracking using multiphoton microscopy in a rat survival surgery model: a promising pre-clinical study for enhanced nerve-sparing surgery. BJU Int. 2015;116:478-86.

18. Tewari AK, Shevchuk MM, Sterling J, et al. Multiphoton microscopy for structure identification in human prostate and periprostatic tissue: implications in prostate cancer surgery. BJU Int. 2011;108:1421-9.

19. Yossepowitch O, Briganti A, Eastham JA, et al. Positive surgical margins after radical prostatectomy: a systematic review and contemporary update. Eur Urol. 2014;65:303-13.

20. Gillitzer R, Thuroff C, Fandel T, et al. Intraoperative peripheral frozen sections do not significantly affect prognosis after nervesparing radical prostatectomy for prostate cancer. BJU Int. 2011;107:755-9.

21. Vasdev N, Agarwal S, Rai BP, et al. Intraoperative frozen section of the prostate reduces the risk of positive margin whilst ensuring nerve sparing in patients with intermediate and high-risk prostate cancer undergoing robotic radical prostatectomy: First Reported UK Series. Curr Urol. 2016; 9:93-103. 